2016-01

\title{
Looking ahead: Anticipatory cueing of attention to objects others will look at.
}

Joyce, $\mathrm{K}$

http://hdl.handle.net/10026.1/4178

10.1080/17588928.2015.1053443

Cogn Neurosci

All content in PEARL is protected by copyright law. Author manuscripts are made available in accordance with publisher policies. Please cite only the published version using the details provided on the item record or document. In the absence of an open licence (e.g. Creative Commons), permissions for further reuse of content should be sought from the publisher or author. 
This article was downloaded by: [University of Plymouth]

On: 10 July 2015, At: 01:02

Publisher: Routledge

Informa Ltd Registered in England and Wales Registered Number: 1072954 Registered office: 5 Howick Place, London, SW1P IWG

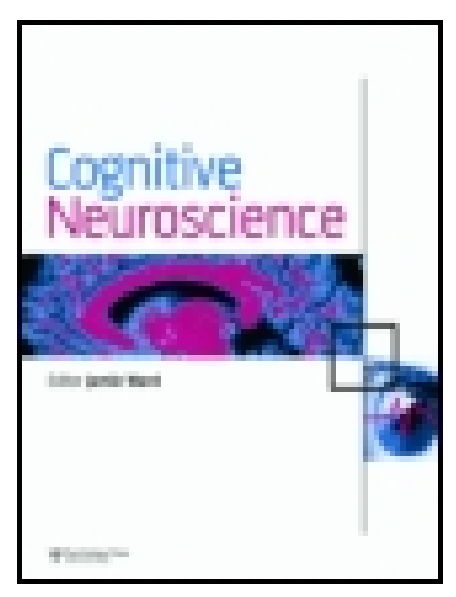

\section{CrossMark}

\section{Cognitive Neuroscience}

Publication details, including instructions for authors and subscription information: http:// www. tandfonline.com/loi/ pens20

\section{Looking ahead: Anticipatory cueing of attention to objects others will look at}

\author{
Katherine J oyce ${ }^{a}$, Kimberley Schenke ${ }^{a}$, Andrew Bayliss ${ }^{b} \&$ Patric Bach $^{a}$ \\ a School of Psychology, Plymouth University, Plymouth, UK \\ b School of Psychology, University of East Anglia, Norwich, UK \\ Published online: $09 \mathrm{~J}$ ul 2015.
}

To cite this article: Katherine J oyce, Kimberley Schenke, Andrew Bayliss \& Patric Bach (2015): Looking ahead: Anticipatory cueing of attention to objects others will look at, Cognitive Neuroscience, DOI: 10.1080/ 17588928.2015.1053443

To link to this article: http:// dx.doi.org/ 10.1080/17588928.2015.1053443

\section{PLEASE SCROLL DOWN FOR ARTICLE}

Taylor \& Francis makes every effort to ensure the accuracy of all the information (the "Content") contained in the publications on our platform. However, Taylor \& Francis, our agents, and our licensors make no representations or warranties whatsoever as to the accuracy, completeness, or suitability for any purpose of the Content. Any opinions and views expressed in this publication are the opinions and views of the authors, and are not the views of or endorsed by Taylor \& Francis. The accuracy of the Content should not be relied upon and should be independently verified with primary sources of information. Taylor and Francis shall not be liable for any losses, actions, claims, proceedings, demands, costs, expenses, damages, and other liabilities whatsoever or howsoever caused arising directly or indirectly in connection with, in relation to or arising out of the use of the Content.

This article may be used for research, teaching, and private study purposes. Any substantial or systematic reproduction, redistribution, reselling, loan, sub-licensing, systematic supply, or distribution in any form to anyone is expressly forbidden. Terms \& Conditions of access and use can be found at http:// www.tandfonline.com/page/terms-and-conditions 


\title{
Looking ahead: Anticipatory cueing of attention to objects others will look at
}

\author{
Katherine Joyce ${ }^{1}$, Kimberley Schenke ${ }^{1}$, Andrew Bayliss ${ }^{2}$, and Patric Bach ${ }^{1}$ \\ ${ }^{1}$ School of Psychology, Plymouth University, Plymouth, UK \\ ${ }^{2}$ School of Psychology, University of East Anglia, Norwich, UK
}

\begin{abstract}
Seeing a face gaze at an object elicits rapid attention shifts toward the same object. We tested whether gaze cueing is predictive: do people shift their attention toward objects others are merely expected to look at? Participants categorized objects while a face either looked at this object, at another object, or straight ahead. Unbeknownst to participants, one face would only look at drinks and the other at foods. We tested whether attention was drawn toward objects "favored" by a face even when currently looking straight ahead. Indeed, while gaze expectations initially had a disruptive effect, participants did shift attention to the faces' favored objects once learning had been established, as long as emotional expressions had indicated personal relevance of the object to the individual. These data support predictive models of social perception, which assume that predictions can drive perception and action, as if these stimuli were directly perceived.
\end{abstract}

Keywords: Gaze cueing; Joint attention; Predictive coding; Prediction; attention.

Humans routinely track what other people know, want, and feel, for a large number of individuals, without being explicitly aware of it (e.g., Schneider, Bayliss, Becker, \& Dux, 2012). A key process in this ability is the human tendency to follow others' gaze. Seeing a face look at an object causes a rapid, spontaneous shift of spatial attention in the observer toward the same target (Driver et al., 1999; see Frischen, Bayliss, \& Tipper, 2007; for review). By synchronizing attention across interaction partners, this "gaze cueing" effect may underpin, at least in part, mankind's remarkable ability for social learning, cooperation, and communication. Conversely, disruptions of this process may underlie some of the social difficulties in autism spectrum and related conditions (Baron-Cohen et al., 1995).

The present study tests whether gaze cueing is predictive. Do we just follow the overt gaze of other people, or do we also routinely shift our attention toward what we expect other people will look at? Everyday behavior is ripe with such anticipatory attention shifts, where people's attention is guided by the relevance an object has, not to themselves, but to their interaction partners. Parents report that their attention is often drawn to objects (diggers, tractors, princesses, and sweets) that their children would like. People helpfully point out objects others are looking for, and, when shopping, they have a knack of finding items that their friends would like.

Such predictive gaze-cueing effects emerge naturally from recent hierarchical feedback models of perception (Clark, 2013; Friston \& Kiebel, 2009; Kilner, Friston, \& Frith, 2007). In such models, perception is not only driven by bottom-up sensory information, but is directly informed by prior knowledge about forthcoming events. In many

Correspondence should be addressed to: Patric Bach, School of Psychology, Plymouth University, Drake Circus, Devon PL4 8AA, UK. E-mail: patric.bach@plymouth.ac.uk

We thank Steven P. Tipper, University of York, for helpful comments during data collection and interpretation.

No potential conflict of interest was reported by the authors.

Patric Bach's work was supported by the Economic and Social Research Council [grant number ES/J019178/1]. 
cases, these predictions can "stand in" for what is actually observed, and induce perceptual biases. For example, people misperceive the disappearance point of a moving object further along the trajectory than it really is (Freyd \& Finke, 1984). These predictions of future motion are guided by one's assumptions about the external forces acting on the object (cf. Hubbard, 2005) and are integrated, in a Bayesian manner, with actual stimulation (e.g., Roach, McGraw, \& Johnston, 2011). In the social domain, it has been shown that heads appear to be rotated further if their gaze suggests a looking goal in this direction (Hudson, Hong-Liu, \& Jellema, 2009). Hands are perceived closer to an object when the observer anticipates a reach and further away if they anticipate a withdrawal (Hudson, Nicholson, Ellis, \& Bach, under review-a, under review-b), and observers more speedily identify actions an individual typically carries out with an object, compared to actions that are equally likely but typical for someone else (Schenke, Wyer, \& Bach, under review).

These findings provide initial evidence that people constantly predict others' future behavior and that these predictions can, to some extent, stand in for sensory information, as if these stimuli were directly perceived. Here, we test, for the first time, whether observers make such predictions about others' gaze, and whether these gaze predictions lead to similar attentional shifts as directly perceived gaze. Prior research has provided suggestive evidence for this idea. For example, gaze cueing can be elicited not only by directly perceived gaze but merely by the belief that a person looks at an object (Teufel, Alexis, Clayton, \& Davis, 2010). Moreover, Frischen and Tipper (2006) have reported that, when re-encountering a face one has seen before, attention is guided not only by the face's current gaze, but by its gaze in the previous encounter. Frischen and Tipper interpreted this as a recall of the observer's own prior attentional state, but it might also reflect an anticipatory coding of gaze (cf. Bayliss \& Tipper, 2006). The person in the photograph had been looking left so we predict the photograph to show the same gaze when seen again.

To test whether gaze expectations can induce such anticipatory gaze cueing, we adapted the standard gaze-cueing paradigm. In each trial, participants saw one of two faces in the middle of two objects. A target appeared on one of the objects and participants indicated, in a speeded response, whether the object was a food or a drink. In half the trials, the face would gaze-and smile - at one of the two objects just prior to the cue. Participants were instructed to ignore these gaze shifts, and we manipulated, unbeknownst to participants, which objects the two faces would look at. One face would only look and smile at drinks, and the other at foods. According to prior work, these gaze shifts should involuntarily direct attention to the gazed-at object, rendering it easier to identify.

The key question is whether people form gaze expectations about the two individuals, such that attention would shift toward their favored object, even when the individuals looked straight ahead. Participants should then more quickly identify objects the face would typically gaze at, compared with objects it typically looks away from, specifically in the second half of the experiment, when participants have learned the relationships between faces and objects. Such an effect could not be explained by a mere reactivation of the observer's prior attentional state. Overall, both faces equally often looked left and right, and their favored objects were seen equally often in both locations. Finding gazecueing effects toward the faces' favored objects would therefore reveal an anticipatory cueing of attention guided by predictions about the other individuals' behavior.

\section{METHOD}

\section{Participants}

Sixty-two students from Plymouth University (1852 years, 14 male) took part. Sample size was determined by a power analysis conducted on pilot data (see Supplementary material). For half of the participants the presented faces were cartoon faces and for the other half the presented faces were real face photographs. Cartoon faces are typically more effective in evoking gaze shifts, most likely because their visual simplicity facilitates cue extraction (e.g., Hietanen \& Leppänen, 2003) and are therefore the most powerful test of the experimental hypothesis. Face photographs, in contrast, allow us to test whether any effects generalize to more realistic face representations that more plausibly represent different individuals. Two participants, one from each group, were excluded because they made more than $10 \%$ errors. The study was approved by the ethics committee of Plymouth University, and adhered to the ethical guidelines of the ESRC, in accord with the declaration of Helsinki. 


\section{Material and apparatus}

All participants completed the Autism Quotient (AQ; Baron-Cohen, Wheelwright, Skinner, Martin, \& Clubley, 2001). The experiment proper was administered via Presentation software, on a Windows XP SP3 $1280 \times 1024$ 32-bit color 17" display. Stimuli were assembled from 25 images: a fixation cross (the "+" symbol in Microsoft's Trebuchet font), four color photographs each of foods (orange, cupcake, apple, and hotdog) and drinks (cola can, orange juice, milkshake, and coffee) and cartoon and photograph versions of a male and a female face. The cartoon faces were generated with MS PowerPoint and the face photographs were taken from the Radboud Face Database (Langner, Dotsch, Bijlstra, Wigboldus, Hawk, \& Van Knippenberg, 2010). The faces could either look straight ahead with a neutral expression, blink (eyes closed) with a neutral expression, or smile while looking left or right (Figure 1).

\section{Design and procedure}

The participants were seated roughly $60 \mathrm{~cm}$ away from the monitor. They filled out the Autism Quotient, were given verbal and on-screen instructions, and then completed 16 practice trials. The experiment proper consisted of 256 trials. Participants were allowed to press "p" at any point to take a break.

Each trial started with a fixation cross $(400 \mathrm{~ms})$. After a brief blank $(600 \mathrm{~ms})$, one of two faces appeared in the middle of the screen, with a food item and a drink on either side (locations counterbalanced across trials). The face looked straight ahead with a neutral expression for a random time interval between 500 and $1100 \mathrm{~ms}$ and then blinked for $100 \mathrm{~ms}$. The next image showed the face with eyes open, in $50 \%$ of the trials looking straight ahead with a neutral expression, or smiling and looking at one of the objects in the other $50 \%$ of the trails. One face would always look at the drinks and never at foods, while the other face showed the opposite behavior (counterbalanced between participants). After $400 \mathrm{~ms}$, a blue square appeared on one object and participants categorized this object as either a drink or a food by pressing either " $\mathrm{h}$ " or the space bar. This image was presented until participants made a response or a maximum trial time of four seconds had passed. Feedback was given for error and timeouts reminding participants of the key assignment.
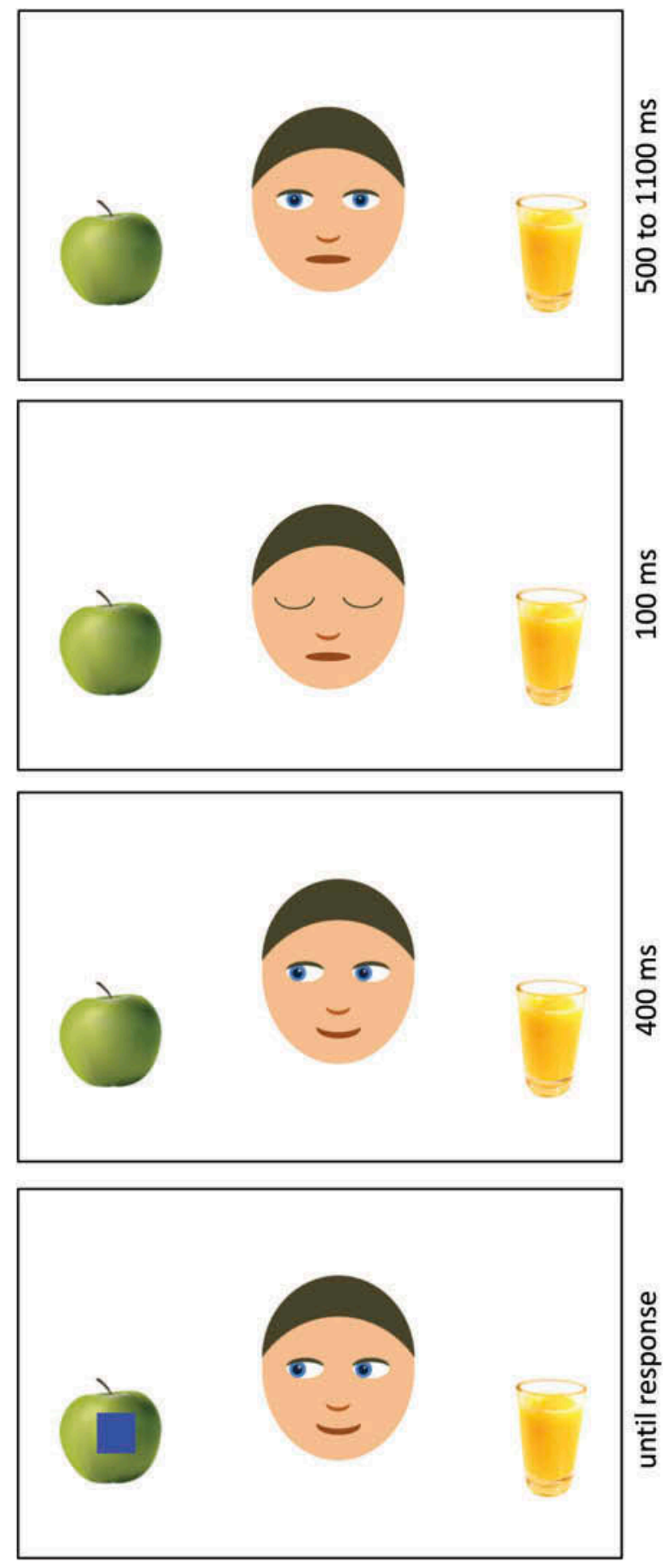

Figure 1. Schematic of the trial sequence. After a fixation cross $(400 \mathrm{~ms})$ and a blank screen $(600 \mathrm{~ms})$, participants saw either a male or female face flanked by a drink and a food item. After a random time interval (between 500 and $1100 \mathrm{~ms}$ ), the face blinked (for $100 \mathrm{~ms}$ ). When the eyes opened again, the face either looked straight ahead (not shown) or at one of the two objects, and a blue square marking the object to be categorized appeared $400 \mathrm{~ms}$ later. We manipulated the gaze behavior of the two faces such that, if looking sideways, one face would only look at drinks and the other only at food (counterbalanced between participants). 
After the experiment, participants were asked whether they noticed a pattern in the stimuli; none did, and several expressed surprise when the manipulation was revealed.

\section{RESULTS}

As during piloting (Supplementary material), trials (1.7\% in total) were excluded if they contained: responses before or after the response interval, responses with uncertain presentation timing (> $10 \mathrm{~ms}$ ), or responses with RTs (response times) \pm 3 standard deviations from the participant's mean in the straight or sideways gaze trials. As participants initiated pauses themselves, trials following or preceding pauses were also excluded. Participants with AQ scores in the clinical range ( $>32$ ) would have been excluded, though none were excluded.

Data were analyzed separately for the gaze-toward object and gaze-straight trials. The gaze trials tested whether our paradigm evoked the expected gazecueing effects, but do not say anything about gaze expectancies, as the faces looked at their favored object in all of these trials. In contrast, the trials with straight gaze measured gaze expectancies, and whether attention was directed toward the favored object even when the face looked straight ahead.

\section{Regular gaze cueing}

We first confirmed that our paradigm elicited the typical gaze-cueing effects. We analyzed the gazetoward object trials with a 2 X 2 mixed-factors ANOVA with Object (looked at, not looked at) and Block $(1,2)$ as repeated measures factors and Group (cartoon faces, photographs) as the between-subjects factor. The analysis of RTs (Table 1) only revealed a main effect of Object $F(1,58)=90.8, M S E=1563$; $p<.001, \eta_{p}{ }^{2}=0.610$, but no other effects $\left(F_{\mathrm{S}}<1\right)$. Participants more quickly categorized a looked-at object than a not looked-at object, in both Block 1,

TABLE 1

Regular gaze-cueing effects for both response times and error rates. Values in brackets show the standard deviation in the condition

\begin{tabular}{llllll}
\hline & \multicolumn{2}{c}{ Response times $(\mathrm{ms})$} & & \multicolumn{2}{c}{ Error rates (\%) } \\
\cline { 2 - 3 } \cline { 5 - 6 } Group & looked at & not looked at & & looked at & not looked at \\
\hline Cartoons & $509(83)$ & $562(89)$ & & $3.3(2.9)$ & $5.2(3.6)$ \\
Photographs & $544(116)$ & $587(114)$ & & $4.4(3.8)$ & $2.8(2.5)$ \\
\hline
\end{tabular}

$\left.F(1,58)=54.7 ; M S E=1248 ; p<0.001, \eta_{p}{ }^{2}=0.486\right)$ and Block 2, $F(1,58)=58.6 ; M S E=1259 ; p<.001$, $\left.\eta_{p}{ }^{2}=0.502\right)$. The analogous analysis of error rates (Table 1$)$ revealed no effects $\left(F_{\mathrm{S}}<1\right)$ except for an interaction of Object by Group, $F(1,58)=13.3$; $p<.001, M S E=0.019 ; \eta_{p}{ }^{2}=0.19$. For cartoon faces, participants made more errors for not lookedat-objects and for photographs they made more errors for looked-at objects (Table 1).

\section{Anticipatory gaze cueing}

The crucial test was whether observers would also shift their attention to the expected object when the face looked straight ahead. RTs and Error rates in the straight-gaze trials were analyzed with the same ANOVA as the regular-gaze trials, with the Object factor now coding whether the target appeared on the object the face typically looked at, or the other object.

The analysis of RTs (Figure 2, left panels) revealed neither an effect of Object, $F<1$, nor of Block, $F$ $(1,58)=3.5, M S E=2052, p=.066, \eta_{p}{ }^{2}=0.057$, but a significant interaction between both factors, $F$ $(1,58)=12.116, M S E=761, p=.001, \eta_{p}{ }^{2}=0.173$. Step down ANOVAs revealed that, in Block 1, participants were faster to categorize typically looked away from objects compared to typically looked-at objects, $F(1,58)=6.2, M S E=822$, $p=.015, \eta_{p}{ }^{2}=0.097$, while Block 2 revealed the expected pattern of faster responses for objects the person typically looks at, $F(1,58)=4.9, M S E=834$, $p=.031, \eta_{p}{ }^{2}=0.078$. Group did not interact with any factor $(F \mathrm{~s}<1)$. The Object by Block interaction was replicated in the photograph group, $F(1,29)=4.6$, $M S E=874, p=.040, \eta_{p}{ }^{2}=0.138$, and the cartoon group $F(1,29)=8.0, M S E=647, p=.008$, $\eta_{p}{ }^{2}=0.217$, when analyzed separately (Figure 2 ).

The analysis of error rates (Figure 2, right panels) revealed a significant effect of Block $F(1,58)=6.7$, $M S E=0.001, p=.012, \eta_{p}{ }^{2}=0.104$, with fewer errors in the second half of the experiment. Importantly, there was a significant effect of Object, $F$ $(1,58)=4.7, M S E=0.001, p=.034, \eta_{p}{ }^{2}=0.075$, but no interaction, $F<1$. Step-down ANOVAs revealed that participants made fewer errors for typically gazed-at objects in Block 2, $F$ $(1,58)=4.863, M S E=0.001, p=.031$, $\eta_{p}{ }^{2}=0.077$, but not in Block $1, F(1,58)=1.065$, $M S E=0.001, p=.306, \eta_{p}{ }^{2}=0.18$. Group did not interact with any other factor $\left(F_{\mathrm{S}}<2.7\right)$.

A separate study (see Supplementary material) tested whether the same effects were also obtained when the observed faces did not smile but showed a neutral 

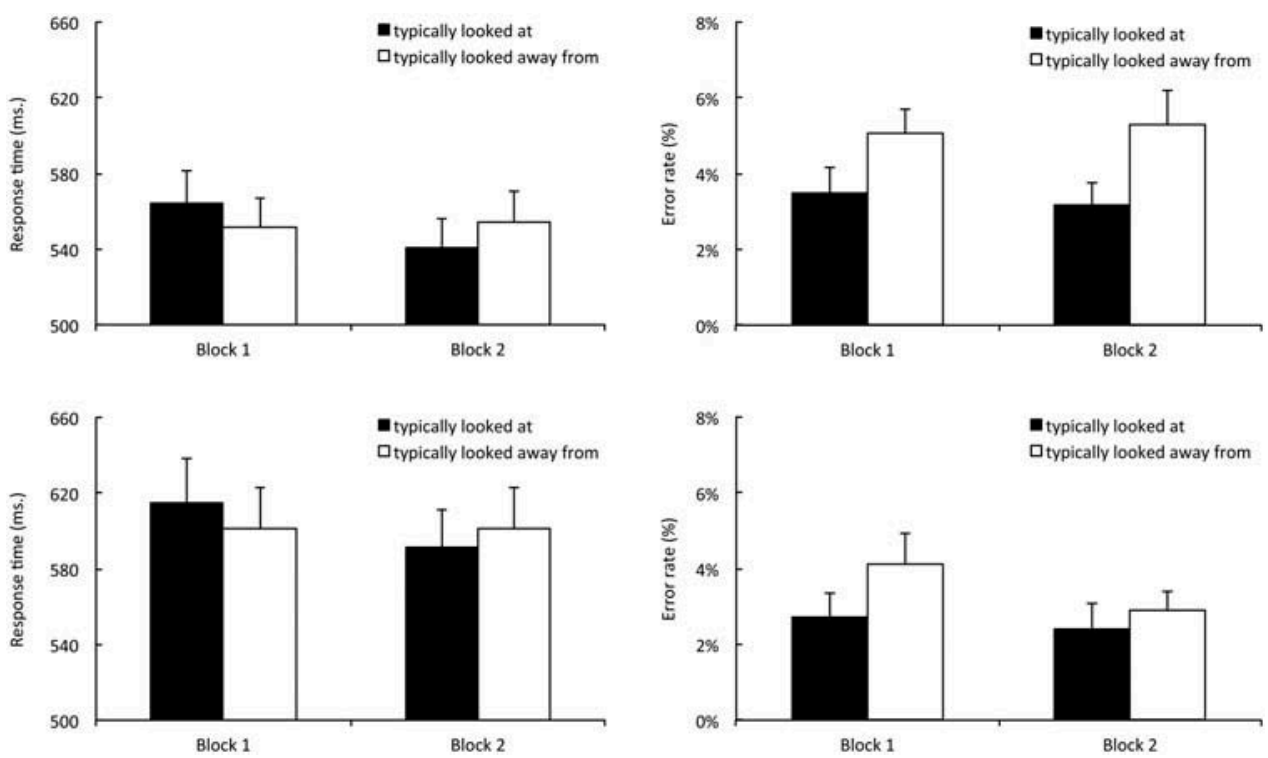

Figure 2. Response times (left panels) and error rates (right panels) in the straight-gaze trials, for the cartoon faces (top row) and real faces (bottom rows) groups separately. In each figure, the left two bars show the data for the first half of the experiment, and the right two bars show the data for the second half of the experiment. The black bars show categorization response for objects that are typically looked at by the shown individual, and the white bars show objects that this individual typically looks away form. Error bars show the standard error of the mean.

expression when looking at the objects, but was otherwise identical. While this study replicated the regular gaze-cueing effects, with faster responses for looked-at compared to not looked-at objects, all predictive gaze-cueing effects in both RTs and Error Rates were eliminated, all $F \mathrm{~s}<1.1$. Indeed, acrossexperiment comparisons revealed that the predictive gaze-cueing effects in the main experiment differed significantly from this control experiment, both for the RT interaction, $F(1,118)=10.5, M S E=722, p<.002$, $\eta_{p}{ }^{2}=0.082$, and the error rate effect in Block $2, F$ $(1,118)=4.150, M S E=0.004, p<.044, \eta_{p}{ }^{2}=0.034$.

\section{GENERAL DISCUSSION}

We tested whether gaze cueing of attention is driven only by bottom-up gaze cues, or whether it is predictive, and is guided by the gaze a person is only expected to show. Participants categorized objects while a face either looked at this object, at another object, or straight ahead. Unbeknownst to participants, we manipulated the looking behavior of the faces, such that one face (e.g., the male) would only look at foods and the other (e.g., the female) would only look at drinks. We tested whether participants would derive implicit gaze expectations from these contingencies, and whether these expectations would elicit similar shifts of attention even when the faces looked straight ahead.
As predicted, the second half of the experiment, after participants had learned the individuals' looking behaviors, revealed such anticipatory cueing effects. Participants categorized objects more quickly and reliably if these objects were typically looked and smiled at by the individual, compared to the objects that they typically looked away from. These anticipatory shifts of attention were observed even though (1) neither the face nor gaze direction was task relevant; (2) gaze direction did not predict the location of the task-relevant object; and (3) across faces, all objects were looked at equally often. The data therefore indicate that, by Block 2, observers had implicitly formed internal models of the individuals' gaze behavior, which then directed attention toward the expected object, as if the gaze shift were directly observed. Gaze cueing of attention is therefore not only driven by bottom-up sensory information, but also by expectations about others' gaze, supporting the assumption that predicted stimulation can drive action and cognition, as if the event was directly observed (cf. Clark, 2013; Friston \& Kiebel, 2009).

We had expected that these effects would slowly build up, and that anticipatory gaze cueing was small or absent in the first half of the experiment. However, an unexpected pattern emerged in these earlier trials. Participants were slower to identify objects that the other person typically looked at, compared to objects they typically looked away from. The shift from negative to positive gaze-cueing effects over the 
experiment was replicated in both the face photograph and cartoon faces groups. It suggests that participants learn about the predictive relationships between individuals, objects, and gaze very quickly. At the start, these predictions have a disruptive effect. Only when fully established do they guide attention similarly as directly observed gaze.

Although not predicted, such negative effects are in line with hierarchical feedback models of perception. These models conceptualize perception as an integration of sensory information and top-down predictions, such that predictions can "stand in" for what was actually perceived (Clark, 2013; Friston \& Kiebel, 2009). These assimilative effects of prior information are, however, typically only observed when the stimuli are not directly attended (Kok, Rahnev, Jehee, Lau, \& de Lange, 2011). Attended stimuli may cause reverse effects and bias perception away from the predictions. Classic examples are visual after-images, where observing — or even imagining - a movement to the left causes subsequent static images to be perceived as moving subtly to the right (Wade, Thompson, \& Morgan, 2014), and we have observed similar contrastive effects in motor activation when famous athletes were seen not performing their typical sports (Bach \& Tipper, 2006; Tipper \& Bach, 2011). In the same way, here, the negative cueing effects might emerge because the individual's straight gaze is coded relative to the expected looking behavior, causing a bias in the opposite direction. When, however, participants become more effective at diverting attention away from the distracting faces over the course of the experiment, these effects revert to positive ones, revealing the expected superposition of sensory information and prior expectations. Of course, at the moment, these considerations are speculative and need to be further verified.

An interesting observation was that the predictive gaze-cueing effects depended on the faces showing an emotional response when looking at the objects (see also Bayliss, Griffiths, \& Tipper, 2009; Bayliss, Schuch, \& Tipper, 2010). In a separate experiment (Supplementary material) - initially used for piloting, but increased to full sample size at the request of a reviewer-we ran exactly the same procedure, but with the faces showing a neutral expression when looking at their objects. While the regular gaze-cueing effects were identical across experiments, all predictive gaze-cueing effects were now eliminated. This difference is striking given that predictive coding models do not necessarily distinguish between social and other effects on perception. This dependency on an emotional expression reveals a social coding of the individuals' gaze, reflecting, perhaps, the mental states that the emotional expressions imply, or the emotional relevance the object has to the individuals (cf. Manera, Elena, Bayliss, \& Becchio, 2014; Bach, Nicholson, \& Hudson, 2014). It is in line with the idea that internal models of other people do not only reflect their behaviors toward objects, but also the internal states these behaviors imply (Barresi \& Moore, 1996). Our new data therefore provide the first evidence for models suggesting that such top-down knowledge can be converted into concrete predictions of others' gaze that drives one's own attention system as if the gaze shifts were directly observed, as predicted by predictive coding of social perception (e.g., Kilner, Friston, \& Frith, 2007).

An important question is whether the predictive effects on gaze cueing reflect explicit or implicit learning of the contingencies. This question was not central to the current hypotheses, but in a postexperiment screening none of the participants reported awareness of the patterns. This unawareness probably emerged because the faces of the individuals were task irrelevant and there was no relationship between gazed-at-object and object-to-be-categorized. Indeed, many participants stated that they attempted to ignore the faces altogether. Moreover, in a prior study, we showed that participants often remain unaware of similar relationships between individuals, situations, and behaviors (Schenke et al., under review), even when alerted, several times during the experiment, that such a pattern was present. Together with the reports of our participants, these data indicate that the typical behavior of others is learned in a largely implicit manner, but nevertheless directly affects perception and guidance of attention.

\section{CONCLUSIONS}

Gaze cueing of attention is not only driven by bottom-up sensory information, but also by prior expectations about what other individuals will look at in a given situation. Because gaze cueing is central for joint attention, learning from others and fluent social interaction, such a predictive mechanism could have far-reaching impacts on social interaction, allowing people to attend to what others will look at in the future, or direct their attention to objects that are relevant to their interaction partners. Future experiments need to explore the role of the implicit/explicit nature of the predictions, and why, in 
the first half of the experiment, predictions can give rise to paradoxical negative gaze-cueing effects.

\section{Supplementary material}

Supplementary (Figure/Table/content) is available via the "Supplementary" tab on the article's online page (http://dx.doi.org/10.1080/17588928.2015.1053443)

For access to data and stimuli, please contact the corresponding author, patric.bach@plymouth.ac.uk

Original manuscript received 1 December 2014

Revised manuscript received 7 April 2015

First published online 24 June 2015

\section{REFERENCES}

Bach, P., \& Tipper, S. P. (2006). Bend it like Beckham: Embodying the motor skills of famous athletes. The Quarterly Journal of Experimental Psychology, 59(12), 2033-2039. doi:10.1080/17470210600917801

Bach, P., Nicholson, T., \& Hudson, M. (2014). The affordance-matching hypothesis: how objects guide action understanding and prediction. Frontiers in Human Neuroscience, 8. doi:10.3389/fnhum.2014.00254

Baron-Cohen, S., Campbell, R., Karmiloff-Smith, A., Grant, J., \& Walker, J. (1995). Are children with autism blind to the mentalistic significance of the eyes? British Journal of Developmental Psychology, 13(4), 379-398. doi:10.1111/bjdp.1995.13.issue-4

Baron-Cohen, S., Wheelwright, S., Skinner, R., Martin, J., \& Clubley, E. (2001). The autism-spectrum quotient (AQ): Evidence from Asperger syndrome/highfunctioning autism males and females, scientists and mathematicians. Journal of Autism and Developmental Disorders, 31(1), 5-17. doi:10.1023/A:1005653411471

Barresi, J., \& Moore, C. (1996). Intentional relations and social understanding. Behavioral and Brain Sciences, 19, 107-154.

Bayliss, A. P., Griffiths, D., \& Tipper, S. P. (2009). Predictive gaze cues affect face evaluations: The effect of facial emotion. European Journal of Cognitive Psychology, 21(7), 1072-1084. doi:10.1080/ 09541440802553490

Bayliss, A. P., Schuch, S. E., \& Tipper, S. P. (2010). Gaze cueing elicited by emotional faces is influenced by affective context. Visual Cognition, 18(8), 1214-1232. doi:10.1080/13506285.2010.484657

Bayliss, A. P., \& Tipper, S. P. (2006). Gaze cues evoke both spatial and object-centered shifts of attention. Perception \& Psychophysics, 68(2), 310-318. doi:10.3758/ BF03193678

Clark, A. (2013). Whatever next? Predictive brains, situated agents, and the future of cognitive science. Behavioral and Brain Sciences, 36, 1-73. doi:10.1017/S0140525X1 2000477
Driver, J., Davis, G., Ricciardelli, P., Kidd, P., Maxwell, E., \& Baron-Cohen, S. (1999). Gaze perception triggers reflexive visuospatial orienting. Visual Cognition, 6(5), 509-540. doi:10.1080/135062899394920

Freyd, J. J., \& Finke, R. A. (1984). Representational momentum. Journal of Experimental Psychology: Learning Memory and Cognition, 10, 126-132.

Frischen, A., Bayliss, A. P., \& Tipper, S. P. (2007). Gaze cueing of attention: Visual attention, social cognition, and individual differences. Psychological Bulletin, 133 (4), 694-724. doi:10.1037/0033-2909.133.4.694

Frischen, A., \& Tipper, S. P. (2006). Long-term gaze cueing effects: Evidence for retrieval of prior states of attention from memory. Visual Cognition, 14(3), 351-364. doi:10.1080/13506280544000192

Friston, K. J., \& Kiebel, S. (2009). Cortical circuits for perceptual inference. Neural Networks, 22, 1093-1104. doi:10.1016/j.neunet.2009.07.023

Hietanen, J. K., \& Leppänen, J. M. (2003). Does facial expression affect attention orienting by gaze direction cues? Journal of Experimental Psychology: Human Perception and Performance, 29(6), 1228.

Hubbard, T. L. (2005). Representational momentum and related displacements in spatial memory: A review of the findings. Psychonomic Bulletin \& Review, 12, 822851. doi:10.3758/BF03196775

Hudson, M., Hong-Liu, C., \& Jellema, T. (2009). Anticipating intentional actions: The effect of eye gaze direction on the judgment of head rotation. Cognition, 112, 423-434. doi:10.1016/j.cognition.2009.06.011

Hudson, M., Nicholson, T., Ellis, R., \& Bach, P. (under review-a). I see what you say: Prior knowledge of other's goals automatically biases the perception of their actions.

Hudson, M., Nicholson, T., Ellis, R., \& Bach, P. (under review-b). One step ahead: the perceived kinematics of others' actions are biased towards expected goals.

Kilner, J. M., Friston, K. J., \& Frith, C. D. (2007). Predictive coding: An account of the mirror neuron system. Cognitive Processing, 8, 159-166. doi:10.1007/s10339-007-0170-2

Kok, P., Rahnev, D., Jehee, J. F., Lau, H. C., \& de Lange, F. P. (2011). Attention reverses the effect of prediction in silencing sensory signals. Cerebral Cortex, bhr310. Cerebral Cortex, 22(9), 2197-2206.

Langner, O., Dotsch, R., Bijlstra, G., Wigboldus, D. H., Hawk, S. T., \& Van Knippenberg, A. (2010). Presentation and validation of the Radboud Faces Database. Cognition and Emotion, 24(8), 1377-1388. doi:10.1080/02699930903485076

Manera, V., Elena, M. R., Bayliss, A. P., \& Becchio, C. (2014). When seeing is more than looking: Intentional gaze modulates object desirability. Emotion, 14(4), 824832. doi:10.1037/a0036258

Roach, N. W., McGraw, P. V., \& Johnston, A. (2011). Visual motion induces a forward prediction of spatial pattern. Current Biology, 21(9), 740-745. doi:10.1016/j. cub.2011.03.031

Schenke, K., Wyer, N., \& Bach, P. (under review). The things you do: implicit person models guide online action observation.

Schneider, D., Bayliss, A. P., Becker, S. I., \& Dux, P. E. (2012). Eye movements reveal sustained implicit 
processing of others' mental states. Journal of Experimental Psychology: General, 141(3), 433-438. doi:10.1037/a0025458

Teufel, C., Alexis, D. M., Clayton, N. S., \& Davis, G. (2010). Mental-state attribution drives rapid, reflexive gaze following. Attention, Perception, \& Psychophysics, 72(3), 695-705. doi:10.3758/APP.72.3.695
Tipper, S. P., \& Bach, P. (2011). The face inhibition effect: Social contrast or motor competition? Journal of Cognitive Psychology, 23(1), 45-51. doi:10.1080/ 20445911.2011.451888

Wade, N. J., Thompson, P., \& Morgan, M. (2014). The after-effect of Adolf Wohlgemuth's seen motion. Perception, 43, 229-234. doi:10.1068/p4304ed 